\title{
Abbreviations, symbols and conventions
}

\section{Conventions}

All phonological representations are rendered in IPA characters and may or may not be enclosed in forward slashes / . . / . Phonetic forms are enclosed in square brackets [. . . . The main line of example sentences is transcribed in the Ngardi orthography and is plain (non-italicised) text. Italics appearing in the main line of example sentences are reserved for loan words or borrowings, for example tayimap=ma-nani 'tie_up=GET-IPFV.PST'. Italics are also used for citing lexemes within text, for example mangarri /manari/ 'vegetable food'.

Suffixal (derivational and inflectional) boundaries in morphologically complex words are indicated by a dash '-'; preverbs which are compounded with a verb in a complex verb construction are represented with an equal sign '=' (e.g. parli=pi-nya 'find=HIT-PST'), which is also used to indicate clitic boundaries (e.g. ya-nta=lu 'GOIMP=PL.S'). Inflecting verb roots are glossed in small caps. Preverbs are glossed with no caps. Portmanteau forms are indicated by a period '. in the glossing line.

Ungrammatical forms are indicated by an asterisk ‘ ', while translations which are uncertain are indicated by an initial question mark '?'

\section{Abbreviations}

Abbreviations used in this work follow the Leipzig glossing conventions (Comrie, Haspelmath \& Bickel, 2008), augmented by additional abbreviations as required.

$\begin{array}{llll}\text { A } & \text { transitive subject } & \text { IPFV } & \text { imperfective } \\ \text { ABS } & \text { absolutive } & \text { IRR } & \text { irrealis } \\ \text { ABL } & \text { ablative } & \text { LCT } & \text { locational bound pronoun } \\ \text { ACCOMP } & \text { accompaniment } & \text { M } & \text { middle voice } \\ \text { ACS } & \text { accessory } & \text { MOVE\&X } & \text { prior associated motion } \\ \text { ADJ } & \text { adjectiviser } & \text { NARR } & \text { narrative } \\ \text { AFF } & \text { affirmative } & \text { NEG } & \text { negative } \\ \text { AGENT } & \text { agentive } & \text { NMLZ } & \text { nominaliser } \\ \text { ALL } & \text { allative } & \text { NPST } & \text { non-past } \\ \text { AM } & \text { associated motion } & \text { O } & \text { object/oblique (pronominal) } \\ \text { ANAPH } & \text { anaphoric propositus } & \text { O } & \text { object of transitive clause } \\ \text { ANTICIP } & \text { anticipatory } & \text { OBL } & \text { oblique (pronominal) } \\ \text { ASRT } & \text { assertive } & \text { OBLIG } & \text { obligative } \\ \text { ASSOC } & \text { associative } & \text { OBV } & \text { obviative } \\ \text { ASSUMP } & \text { assumptive } & \text { PAUCAL } & \text { paucal } \\ \text { ATT } & \text { attenuative } & \text { PERL } & \text { perlative } \\ \text { AUX } & \text { auxiliary } & \text { PFV } & \text { perfective } \\ \text { C, CAT } & \text { catalyst } & \text { PL } & \text { plural }\end{array}$




\begin{tabular}{|c|c|c|c|}
\hline CERT & certainty (particle) & PM & possessum \\
\hline COMP & complementiser & $\mathrm{PO}$ & primary object \\
\hline COMPL & completive & Poss & possessive \\
\hline COMPAR & comparative & РОт & potential \\
\hline CONDIT & conditional & PP & possessive phrase \\
\hline CONJ & conjunction & PPOSS & personal possessive \\
\hline CONTR & contrastive & $P R$ & possessor \\
\hline $\mathrm{CF}$ & counterfactual & PRIV & privative \\
\hline CUST & customary & PROP & proprietive \\
\hline CVC & complex verb construction & PRS & present \\
\hline CM & conjugation marker & PRS.AUX & present auxiliary \\
\hline D.ABL & directional ablative & PRST & presentative \\
\hline DAT & dative & PRTL & presentational \\
\hline DELIM & delimitive & PST & past \\
\hline DEN & denizen & QUOT & quotative \\
\hline DIM & diminutive & RECIP & reciprocal \\
\hline DIR & directional & RECOG & recognitional \\
\hline DU & dual (pronominal) & REFL & reflexive \\
\hline DUAL & dual (nominal) & REP & repetitive \\
\hline DUB & dubitative & RESTR & restrictive \\
\hline DYAD & dyadic & RESULT & resultative \\
\hline EMPH & emphatic & RDP & reduplicant \\
\hline EMPH.AUX & emphatic auxiliary & $\mathrm{S}$ & subject (pronominal) \\
\hline ERG & ergative & $\mathrm{S}$ & intransitive subject \\
\hline ELA & elative & SEMBL & semblative \\
\hline EP & epenthetic & SEQ.AUX & sequential auxiliary \\
\hline EVIT & evitative & SEQLOC & sequential locative \\
\hline EXCL & exclusive & SG & singular \\
\hline HITH & hither & so & secondary object \\
\hline HORT & hortative & SSUB & simultaneous subordinator \\
\hline HYP & hypothetical & SUB & subordinator \\
\hline IMP & imperative & SVC & serial verb construction \\
\hline INCEP & inceptive & $\mathrm{T}$ & temporal subordinator \\
\hline INCL & inclusive & TERM & terminative \\
\hline INCRE & increment & TOP.AUX & topic auxiliary \\
\hline INF & infinitive & TR & transitive \\
\hline INTENS & intensifier & TWD & toward \\
\hline INHAB & inhabitant & UNCERT & uncertain \\
\hline IPFV & imperfective & UNREAL & unrealised \\
\hline 10 & indirect object & voc & vocative \\
\hline IRR & irrealis & X\&MOVE & subsequent associated motion \\
\hline KGROUP & kinship group suffix & 1 & first person \\
\hline KPOSS & possessed kin & 2 & second person \\
\hline LACK & lacking & 3 & third person \\
\hline LAT & lative & q & female \\
\hline LOC & locative & $\hat{\sigma}$ & male \\
\hline
\end{tabular}

